\title{
Distinctive chemical and aromatic composition of red wines produced by Saccharomyces cerevisiae co-fermentation with indigenous and commercial non-Saccharomyces strains
}

\author{
Boqin Zhang ${ }^{\mathrm{a}, \mathrm{b}}$, Violeta Ivanova-Petropulos ${ }^{\mathrm{d}}$, Changqing Duan ${ }^{\mathrm{a}, \mathrm{b}}$, Guoliang Yan ${ }^{\mathrm{a}, \mathrm{b}, \mathrm{c}, \text { * }}$ \\ ${ }^{\text {a }}$ Centre for Viticulture and Enology, College of Food Science and Nutritional Engineering, China Agricultural University, Beijing, 100083, China \\ ${ }^{\mathrm{b}}$ Key Laboratory of Viticulture and Enology, Ministry of Agriculture and Rural Affairs, Beijing, 100083, China \\ ${ }^{\mathrm{c}}$ Key Laboratory of Food Bioengineering (China National Light Industry), Beijing, 100083, China \\ d Faculty of Agriculture, University "Goce Delčev" - Štip, Krste Misirkov 10-A, 2000, Štip, Macedonia
}

\section{A R T I C L E I N F O}

\section{Keywords:}

Torulaspora delbrueckii

Lachancea thermotolerans

Mixed fermentation

Indigenous strain

Aroma compounds

\begin{abstract}
A B S T R A C T
The use of Saccharomyces cerevisiae and non-Saccharomyces yeast species as mixed starters has advantages over pure culture fermentation because of increased wine sensory characteristics. The aim of the present study was to evaluate the divergences of wine compositions fermented by indigenous non-Saccharomyces strains (Torulaspora delbrueckii TD12 and Lachancea thermotolerans LT9) or commercial non-Saccharomyces strains (T. delbrueckii Prelude and L. thermotolerans Concerto) combined with $S$. cerevisiae D254, respectively. Results evidenced that although belong to the same species, the content of chemical and aromatic compounds of red wines produced by indigenous and commercial strains was significantly different after alcoholic fermentation (ALF) and malolactic fermentation (MLF). TD12/D254 was characterized with a higher amount of glycerol, ethyl esters, and volatile acids, whilst Prelude/D254 was distinguished by a higher intensity of isoamyl acetate and a lower production of acetic acid. LT9/D254 increased the intensity of higher alcohols, esters, and $\beta$-damascenone compared with Concerto/D254. After MLF, the diversities variation of glycerol and lactic acid were increased, but acetic acid and most volatile compounds were reduced. TD12/D254 obtained better aromatic quality as assessed by calculating the odor activity values (OAVs). Our results highlighted the strain-specificity of non-Saccharomyces strains in shaping the aromatic characteristic of wine, and suggested that more attention should be paid to the strain-specific characteristics when selecting non-Saccharomyces strains to improve aroma diversity and quality of the wine. In this regard, the indigenous strain is a suitable choice because of better adaptation to fermentation conditions and generating typical sensory characteristics specific to the wine region.
\end{abstract}

\section{Introduction}

The fermentation of grape juice into wine is a complex biochemical process, in which yeasts are primarily responsible for the alcoholic fermentation of the juice, including Saccharomyces cerevisiae and nonSaccharomyces yeasts (Jolly, Varela, \& Pretorius, 2014). It is widely accepted that a selected and inoculated strain of $S$. cerevisiae will dominate the fermentation process and produce most of the ethanol and a broad range of aroma-active compounds in wine (Comitini et al., 2011). Nowadays, non-Saccharomyces yeasts received significant attention because they have some desired enological characteristics that are absent in S. cerevisiae, such as producing high levels of aroma compounds (esters, higher alcohols and acids) and producing and secreting several enzymes (esterase, $\beta$-glycosidase, lipase and protease, among others), which can interact with odorless grape precursors and improve wine complexity in controlled fermentation manner (Ciani, Comitini, Mannazzu, \& Domizio, 2010; Domizio et al., 2011; Escribano et al., 2018; Padilla, Gil, \& Manzanares, 2016; van Wyk, Grossmann, Wendland, von Wallbrunn, \& Pretorius, 2019). To data, several non-Saccharomyces yeasts species, such as Torulaspora delbrueckii, Lachancea thermotolerans, Metschnikowia pulcherrima, Pichia kluyveri were commercialized and recommended to be co-inoculated with $S$. cerevisiae to improve the specific organoleptic characteristics of wine (Prior, Bauer, \& Divol, 2019; Roudil et al., 2020). For example, the

\footnotetext{
* Corresponding author. Centre for Viticulture and Enology, College of Food Science and Nutritional Engineering, China Agricultural University, Beijing 100083, China.

E-mail address: glyan@cau.edu.cn (G. Yan).
} 
co-fermentation with $T$. delbrueckii and $S$. cerevisiae can improve major volatile compounds (such as ethyl octanoate, isoamyl acetate, 2-phenylethyl alcohol) and thiols concentration (Benito, 2018; Chen et al., 2018; Puertas, Jiménez, Cantos-Villar, Cantoral, \& Rodríguez, 2017). And, the sequential inoculation of $L$. thermotolerans with $S$. cerevisiae can lead to an enhancement of lactic acid, resulting in the increased acidity of the final wines (Gobbi et al., 2013; Santiago, 2018).

China is a rapidly developing country and has a great potential for improved wine production and consumption. Currently, there are over ten major viticultural areas and each has distinct ecological characteristics. Among them, Xinjiang and Ningxia are the main two historic and major wine regions, however, the hot climate in summer and intense sun exposure in both regions results in red wines with high alcohol content (up to 14-15\% vol), low acidity, and a lack of fruit notes and elegance in aroma (Duan et al., 2018). To solve the problems of less distinctive aroma and low acidity in wines, inoculating commercial non-Saccharomyces strains become increasingly popular in both wine regions, such as commercial T. delbrueckii and L. thermotolerans. However, because of the usually poor fermentation dynamics of non-Saccharomyces yeasts, the application of commercial non-Saccharomyces strains is doubted about the improvement of oenological quality and styles of regional wines (Nisiotou et al., 2018; Rainieri \& Pretorius, 2000). Moreover, highly strain-dependent variability of the same non-Saccharomyces species also leads to the divergences of wine chemical and aroma quality (Binati et al., 2020; Renault et al., 2009; van Breda, Jolly, \& van Wyk, 2013), which further increased the troubles of choosing suitable non-Saccharomyces strains with the aim of improving the quality of regional wines. To address this problem, inoculating indigenous or locally selected wine yeasts with excellent oenological characteristics is encouraged, owing to these yeasts having high environment adaptability, and more importantly, indigenous yeasts are considered as key factors for the 'terroir' characteristics of regional wine (Li et al., 2020; Raymond, Eder, Reynoso, Lauret, \& Rosa, 2017).

In our previous study, two indigenous non-Saccharomyces yeasts T. delbrueckii CVE-TD12 and L. thermotolerans CVE-LT9 with good technological characteristics, including high fermentation speed, high tolerance to $\mathrm{SO}_{2}$ and sugar, and high activity of $\beta$-glucosidase, have been isolated and preliminary evaluated. Hence, in this study, we assess the improvement effects of indigenous strains T. delbrueckii TD12 and L. thermotolerans LT9 co-fermentation with Saccharomyces cerevisiae on red wines in $20 \mathrm{~L}$ fermenter using Cabernet Sauvignon grape as the must, respectively. The same design was carried out in commercial nonSaccharomyces strains ( $T$. delbrueckii Prelude and L. thermotolerans Concerto) mixed cultures to identify the similarities and differences with indigenous non-Saccharomyces strains. The content of major fermentation products and aromatic compounds of the red wine was determined after alcoholic fermentation (ALF) and malolactic fermentation (MLF), respectively. The results are expected to enrich our understanding of the beneficial effects of local non-Saccharomyces strains in improving basic parameters and aroma composition, and shaping regional wine quality.

\section{Materials and methods}

\subsection{Yeast strains}

Two indigenous non-Saccharomyces strains (T. delbrueckii CVE-TD12 and L. thermotolerans CVE-LT9) with desirable physiological properties were used in this study. They were identified by means of 26S rDNARFLP analysis (Kurtzman \& Robnett, 1998) and deposited in the Centre for Viticulture and Enology, China Agricultural University. Two additional commercial non-Saccharomyces strains, Prelude ${ }^{\mathrm{TM}}$ (T. delbrueckii, Chr. Hansen, Denmark) and Concerto ${ }^{\mathrm{TM}}$ (L. thermotolerans, Chr. Hansen, Denmark) were isolated as a single colony and used in co-fermentation with $S$. cerevisiae D254 (Lalvin, France) for comparison. These strains were stored at $-80^{\circ} \mathrm{C}$ in the YPD medium with glycerol ( $20 \% \mathrm{v} / \mathrm{v}$ final concentration).
Mixed fermentations of indigenous and commercial non-Saccharomyces strains with $S$. cerevisiae in $20 \mathrm{~L}$ fermenter.

Multi-starter fermentations were performed by sequential inoculation using indigenous (TD12 and LT9) or commercial (Prelude and Concerto) non-Saccharomyces strains followed by $S$. cerevisiae strain D254 after 2 days. The pure culture of D254 starter was set as the control trials. The inoculum ratio of non-Saccharomyces and Saccharomyces species was 10:1, and the initial active population of non-Saccharomyces and Saccharomyces were $1.0 \times 10^{7} \mathrm{CFU} / \mathrm{mL}$ and $1.0 \times 10^{6} \mathrm{CFU} / \mathrm{mL}$ precultured in pasteurized Cabernet Sauvignon grape juice, respectively (Renault, Coulon, de Revel, Barbe, \& Bely, 2015). Cabernet Sauvignon grapes $(18 \mathrm{~kg}$ ) picked from the Manasi region were added into $20 \mathrm{~L}$ stainless steel fermenter after destemming, crushing, and adding 60 $\mathrm{mg} / \mathrm{L}$ of sulfur dioxide and $30 \mathrm{mg} / \mathrm{L}$ pectinase. The basic parameters of the must were measured as follows: $\mathrm{pH} 3.23,5.21 \mathrm{~g} / \mathrm{L}$ of titratable acid, $253 \mathrm{~g} / \mathrm{L}$ of sugar. Triplicated fermentations were carried out at $20-23{ }^{\circ} \mathrm{C}$ with regular punching skins down to improve extraction. After alcoholic fermentation (sugar content was below $4 \mathrm{~g} / \mathrm{L}$ ), grape pomace was separated out of wine carefully. The wines were transferred to $10 \mathrm{~L}$ glass fermenter for settle for 2 days and started malolactic fermentation by commercial Oenococcus oeni (Viniflora ${ }^{\circledR}$ Oenos, Chr. Hansen) according to the manufacturer's instructions. The samples were taken after alcoholic fermentation and malolactic fermentation, centrifuged, and stored at $-20^{\circ} \mathrm{C}$ for analysis of glucose, fructose, glycerol, ethanol, acetic acid, non-volatile acids and volatile aroma compounds.

\subsection{Analytical techniques}

The polymerase chain reaction (PCR) fingerprinting of indigenous strains (TD12 and LT9) and commercial strains (Prelude and Concerto) used in mixed fermentation trials were examined by RAPD (Random Amplified Polymorphic DNA) analysis, using the primer sequences ( $5^{\prime}$ GCT CGT CGC T-3') according to the method of Michel et al. (2015) with some modifications. Yeast DNA was isolated using TIANamp Yeast DNA Kit (Tiangen, Beijing, China). Typical PCR was performed with $12.5 \mu \mathrm{L}$ Master Mix $2 \times$ (TsingKe, China), $5 \mu \mathrm{L}$ PCR water, $5 \mu \mathrm{L}$ primer (Sangon Biotech, China) and $2.5 \mu \mathrm{L}$ template DNA with a total reaction volume of $25 \mu \mathrm{L}$. PCR parameters were: (i) $94^{\circ} \mathrm{C} / 4 \mathrm{~min}$; (ii) 30 cycles of $94^{\circ} \mathrm{C} / 45 \mathrm{~s}$, $57{ }^{\circ} \mathrm{C} / 45 \mathrm{~s}, 72{ }^{\circ} \mathrm{C} / 1 \mathrm{~min}$; and (iii) $72{ }^{\circ} \mathrm{C} / 10 \mathrm{~min}$, and PCR was carried out using a thermal cycler (C1000 TouchTM, Bio-rad, Singapore). Amplicons were detected using $1 \%$ agarose gel electrophoresis by PowerPac Basic TM (Bio-rad, Singapore).

Glucose, fructose, glycerol, ethanol, acetic acid, and non-volatile acids of the final wines were determined by HPLC (Agilent Technologies, Santa Clara, CA, USA) equipped with an HPX-87H Aminex ionexchange column $(300 \mathrm{~mm} \times 7.8 \mathrm{~mm}$, Bio-Rad Laboratories, Hercules, CA, USA) (Liu, Lu, Duan, \& Yan, 2016). The mobile phase was $5 \mathrm{mmol}$ sulfuric acid. Glucose, fructose, ethanol, and glycerol were detected with a refractive index detector with the column maintained at $45^{\circ} \mathrm{C}$. Acetic acid, malic acid, lactic acid, and succinic acid were detected with a photodiode array detector at $214 \mathrm{~nm}$ with the column maintained at $60{ }^{\circ} \mathrm{C}$. The samples prior to HPLC analysis were filtered through a 0.22 $\mu \mathrm{m}$ membrane filter (Dikma Technologies, Lake Forest, CA, USA).

The aroma compounds of wines after alcoholic and malolactic fermentation were determined by headspace solid-phase micro-extraction coupled with gas chromatography-mass spectrometry (HS-SPMEGC-MS) according to our previous study (Lan et al., 2016; Xu et al., 2015). The identification of the aroma compounds was based on retention indices of reference standards and mass spectra matching in the standard NIST 11 library. For the quantification of these aroma compounds, the relative peak areas of each identified compounds were measured and then compared with the relative peak area of the added internal standard (4-methyl-2-pentanol). Analyses were performed in duplicate. The detailed quantitation information about linear fit, $\mathrm{R}^{2}$ value, linear range, aroma characteristics and aroma type for the volatile compounds used in this study were showed in Supplementary Table 1. 


\subsection{Statistical analysis}

One-way ANOVA using the Duncan test at significance level $P<0.05$ was carried out to uncover statistical differences between the wines produced from the different inoculation protocols. Principal component analysis (PCA) was conducted using volatile compounds (OAV $>0.1$ ) after alcoholic and malolactic fermentation, respectively. Statistical analyses were performed with the IBM SPSS Statistical Package (version 24.0, IBM Corp, NY, USA).

\section{Results and discussion}

\subsection{PCR fingerprinting of commercial and indigenous non-} saccharomyces strains

Inoculation with different wine yeast strains for wine fermentation can influence wine parameters and volatile profiles due to their genetic variations (Casu, Pinu, Fedrizzi, Greenwood, \& Villas-Boas, 2016; Erasmus, Cliff, \& Vuuren, 2004). To distinguish the indigenous and commercial non-Saccharomyces strains at the strain level, the genetic characterizations of TD12 and Prelude, LT9 and Concerto were determined and compared by RAPD analysis, according to Michel et al. (2015). In Fig. 1, there was high length variation between TD12 and Prelude strains, and additional two bands (480 bp and $1050 \mathrm{bp}$ ) appeared in TD12 strain. As to L. thermotolerans strains, a band of 1500 bp appeared in LT9 strain, which was not observed in Concerto strain. These data indicated that although belonging to the same species, T. delbrueckii or L. thermotolerans strains in this study had significant differences in genetic characterizations, which is in good agreement with the results of Renault et al. (2009).

\subsection{Fermentation progress and basic parameters of wine}

The fermentation progress during ALF in the pure or mixed cultures was monitored by must density and presented in Fig. 2. The pure culture of $S$. cerevisiae D254 had the highest fermentation rate and completed ALF within 8 days. In comparison, the duration time of mixed cultures was extended to $12 \mathrm{~d}$. Noticeably, the fermentation activities of T. delbrueckii TD12 and L. thermotolerans LT9 were all higher than those

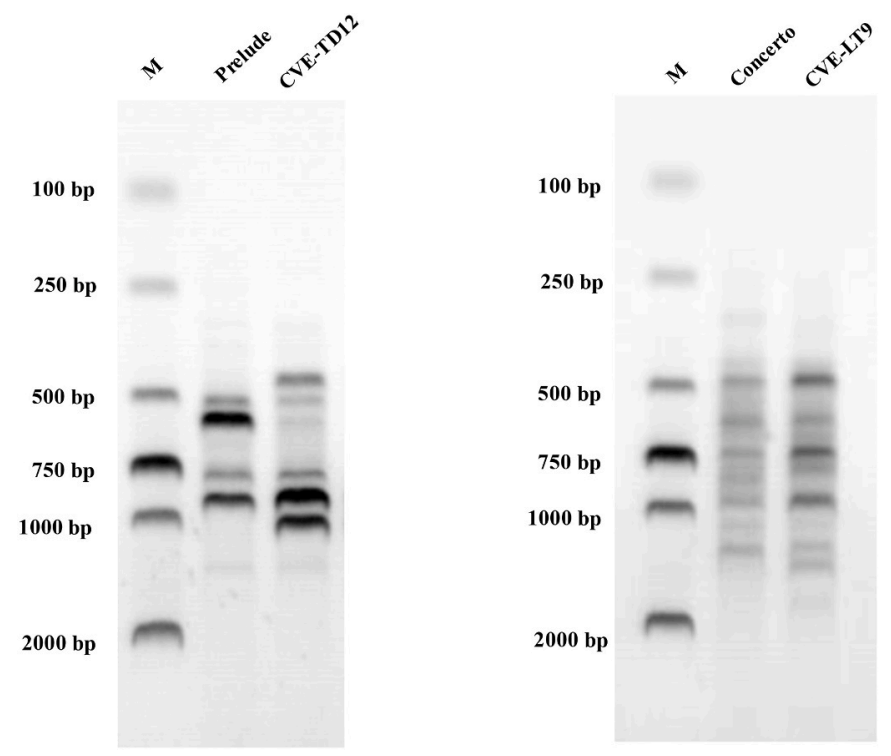

Fig. 1. Electrophoretic patterns of T. delbrueckii and L. thermotolerans strains with $1 \%$ agarose gel resulting from RAPD fingerprint method. T. delbrueckii strains include TD12 and commercial strain Prelude; L. thermotolerans strains include LT9 and commercial strain Concerto. M: 2000 bp DNA ladder.

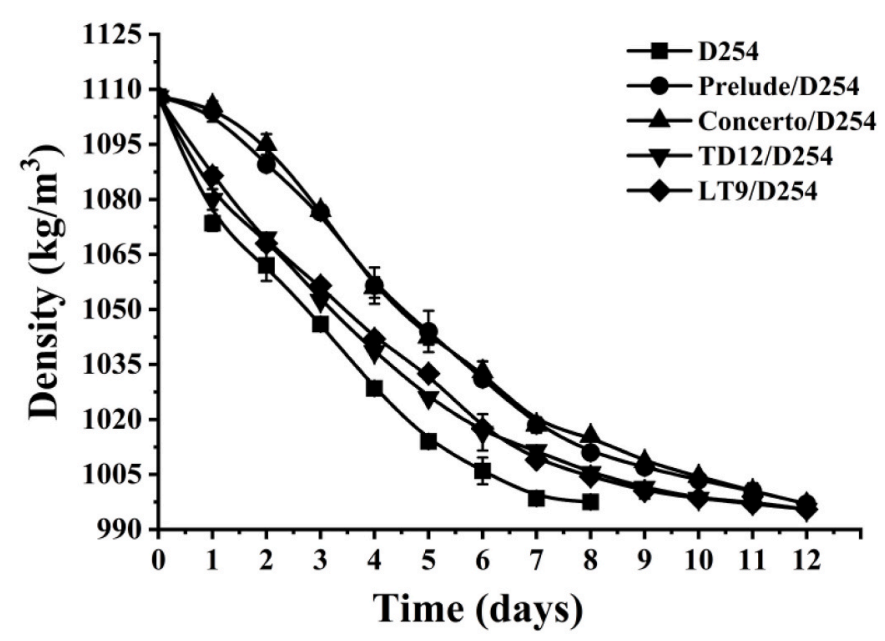

Fig. 2. The fermentation progress of trials inoculated by different strains.

of commercial strains, confirming that indigenous strains can well adapt to grape must composition and environmental conditions of the regional winery (Nisiotou et al., 2018).

Table 1 showed the basic compositions in wines after ALF and MLF. All treatments successfully completed fermentation with total reducing sugar content below $4 \mathrm{~g} / \mathrm{L}$. The production of glycerol was 6.95-9.05 g/ $\mathrm{L}$ after ALF and 8.69-9.73 g/L after MLF, in which TD12/D254 had a significantly increased level. Minor differences of treatments were observed in the case of ethanol concentration after ALF $(P=0.128)$, but the significant diversities appeared after MLF $(P<0.001)$, and the highest values always produced in D254 single fermentation. Acetic acid is a negative fermentation by-product and provides vinegar character to wine with the level above $0.8 \mathrm{~g} / \mathrm{L}$ (Benito, 2018). TD12/D254 and Prelude/D254 that involved $T$. delbrueckii yeasts had a significantly lower production of acetic acid $(0.21 \mathrm{~g} / \mathrm{L})$, which was in consistent with the previous finding that $T$. delbrueckii generated a lower level of acetic acid during winemaking (Canonico, Comitini, \& Ciani, 2017; Chen et al., 2018; Liu, Laaksonen, Kortesniemi, Kalpio, \& Yang, 2018). However, the level of acetic acid after MLF was a range from $0.51 \mathrm{~g} / \mathrm{L}$ to $0.60 \mathrm{~g} / \mathrm{L}$, and no significant differences $(P=0.751)$ were observed in all treatments. Lactic acid usually is perceived as sour and spicy (Vilela, 2019). The obviously higher concentration of lactic acid was produced in wines of Concerto/D254 (1.49 g/L) and LT9/D254 (1.18 g/L), confirmed that L. thermotolerans can increase the lactic acid level and improve the acidity of wine (Balikci, Tanguler, Jolly, \& Erten, 2016; Porter, Divol, \& Setati, 2019). The content of lactic acid further increased after MLF, ranging from $3.02 \mathrm{~g} / \mathrm{L}$ (D254) to $4.58 \mathrm{~g} / \mathrm{L}$ (Concerto/D254). It should be noted that although having less amount after ALF, lactic acid in T. delbruecki/S. cerevisiae (4.25-4.26 g/L) became comparable with L. thermotolerans/S. cerevisiae (4.23-4.58 g/L) after MLF. This implied that the interaction between $T$. delbrueckii strain and lactic acid bacteria might favor lactic acid formation, or specific intermediates produced by T. delbrueckii strain could be transformed to lactic acid. The detailed mechanism needs to be further investigated.

\subsection{Aromatic quality of wines fermented by different yeasts after $A L F$ and $M L F$}

HS-SPME-GC-MS was applied to detect the volatile compounds of red wines inoculated with different $T$. delbrueckii and $L$. thermotolerans strains in this study. Thirty-one aroma compounds in wines after ALF and MLF were identified and quantified (Table 2 and Table 3). The compounds with odor activity value (OAV) greater than one were highlighted, and the compounds of OAV $>0.1$ were underlined.

The higher alcohols were the largest group of aroma compounds in this study, the concentration of $300-400 \mathrm{mg} / \mathrm{L}$ in wine is acceptable, and 
Table 1

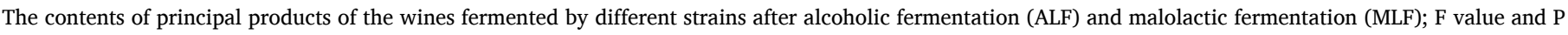
value of main products were analyzed by one-way ANOVA.

\begin{tabular}{|c|c|c|c|c|c|c|c|c|}
\hline & Compounds & D254 & Prelude/D254 & Concerto/D254 & TD12/D254 & LT9/D254 & $\mathrm{F}$ value & $P$ value \\
\hline \multirow[t]{7}{*}{ ALF } & Glucose (g/L) & $0.28 \pm 0.05 b$ & $0.15 \pm 0.01 \mathrm{a}$ & $0.16 \pm 0.04 \mathrm{a}$ & $0.26 \pm 0.06 b$ & $0.28 \pm 0.05 b$ & 7.863 & 0.001 \\
\hline & Fructose $(\mathrm{g} / \mathrm{L})$ & $2.04 \pm 0.24 a$ & $2.04 \pm 0.53 a$ & $2.31 \pm 0.48 \mathrm{ab}$ & $2.72 \pm 0.08 b$ & $2.71 \pm 0.2 b$ & 3.748 & 0.026 \\
\hline & Glycerol (g/L) & $8.12 \pm 0.32 b$ & $7.01 \pm 0.76 a$ & $6.95 \pm 0.22 \mathrm{a}$ & $9.05 \pm 0.2 c$ & $8.1 \pm 0.2 b$ & 19.252 & $<0.001$ \\
\hline & Ethanol (\%v/v) & $14.45 \pm 1.31 \mathrm{~b}$ & $13.62 \pm 0.08 \mathrm{ab}$ & $13.36 \pm 0.4 a$ & $14.23 \pm 0.36 \mathrm{ab}$ & $13.53 \pm 0.29 a b$ & 2.127 & 0.128 \\
\hline & Malic acid (g/L) & $2.35 \pm 0.46 \mathrm{ab}$ & $2.45 \pm 0.38 \mathrm{ab}$ & $2.16 \pm 0.14 \mathrm{a}$ & $3.26 \pm 0.12 c$ & $2.76 \pm 0.08 b$ & 9.260 & 0.001 \\
\hline & Lactic acid (g/L) & $0.66 \pm 0.26 b$ & $0.38 \pm 0.2 \mathrm{a}$ & $1.49 \pm 0.12 \mathrm{~d}$ & $0.49 \pm 0.13 \mathrm{ab}$ & $1.18 \pm 0.06 \mathrm{~b}$ & 32.177 & $<0.001$ \\
\hline & Acetic acid (g/L) & $0.54 \pm 0.08 c$ & $0.21 \pm 0.03 a$ & $0.24 \pm 0.05 a$ & $0.21 \pm 0.02 \mathrm{a}$ & $0.37 \pm 0.06 b$ & 30.512 & $<0.001$ \\
\hline \multirow[t]{5}{*}{ MLF } & Fructose $(\mathrm{g} / \mathrm{L})$ & $1.02 \pm 0.59 \mathrm{a}$ & $0.5 \pm 0.01 \mathrm{a}$ & $0.3 \pm 0.06 \mathrm{a}$ & $0.43 \pm 0.12 \mathrm{a}$ & $0.57 \pm 0.02 \mathrm{a}$ & 2.041 & 0.227 \\
\hline & Glycerol (g/L) & $8.69 \pm 0.16 b$ & $9.01 \pm 0.14 b$ & $7.95 \pm 0.05 a$ & $9.73 \pm 0.13 c$ & $9.53 \pm 0.26 c$ & 37.707 & 0.001 \\
\hline & Ethanol (\%v/v) & $15.32 \pm 0.16 c$ & $15.42 \pm 0.04 c$ & $13.95 \pm 0.06 \mathrm{a}$ & $14.94 \pm 0.13 b$ & $15.26 \pm 0.03 c$ & 75.275 & $<0.001$ \\
\hline & Lactic acid (g/L) & $3.02 \pm 0.12 \mathrm{a}$ & $4.26 \pm 0.01 b$ & $4.58 \pm 0.06 b$ & $4.25 \pm 0.18 b$ & $4.23 \pm 0.23 b$ & 35.248 & 0.001 \\
\hline & Acetic acid (g/L) & $0.57 \pm 0.1 \mathrm{a}$ & $0.51 \pm 0.06 \mathrm{a}$ & $0.51 \pm 0.01 \mathrm{a}$ & $0.56 \pm 0.08 \mathrm{a}$ & $0.6 \pm 0.11 \mathrm{a}$ & 0.481 & 0.751 \\
\hline
\end{tabular}

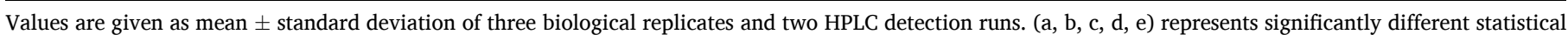
groups $(\mathrm{p}<0.05)$ using the data after alcoholic fermentation and malolactic fermentation, respectively.

Table 2

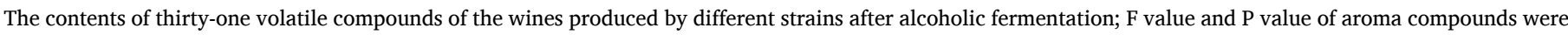
analyzed by one-way ANOVA.

\begin{tabular}{|c|c|c|c|c|c|c|c|}
\hline Compounds $(\mu \mathrm{g} / \mathrm{L})$ & D254 & Prelude/D254 & Concerto/D254 & TD12/D254 & LT9/D254 & F value & $P$ value \\
\hline 1-Hexanol & $\underline{3883.59 \pm 147.13 b}$ & $3245.57 \pm 89.87 a$ & $3265.97 \pm 81.53 a$ & $\underline{4733.83 \pm 115.83 c}$ & $\underline{4542.29 \pm 239.82 \mathrm{c}}$ & 67.538 & $<0.001$ \\
\hline Isobutyl alcohol & $\overline{32239.58 \pm 1334.1 \mathrm{~b}}$ & $\overline{24835.61 \pm 3345.94 a}$ & $\overline{21269.32 \pm 1111.61 a}$ & $\overline{28913.34 \pm 1004.04 \mathrm{~b}}$ & $\overline{24089.12 \pm 2271.68 \mathrm{a}}$ & 13.698 & $<0.001$ \\
\hline Isoamyl alcohol & $\frac{167743.02 \pm}{11572.48 b}$ & $\frac{151842.72 \pm}{10191.81 \mathrm{ab}}$ & $\frac{137589.33 \pm}{8198.61 a}$ & $\frac{197874.98 \pm}{12585.69 c}$ & $\frac{164345.06 \pm}{2475.89 b}$ & 16.028 & $<0.001$ \\
\hline 2,3-Butanediol & $111215.2 \pm 2242.45 \mathrm{~b}$ & $105875.3 \pm 1285.78 \mathrm{a}$ & $114950 \pm 2148.92 b$ & $139531.1 \pm 2563.87 \mathrm{c}$ & $130310.2 \pm 1896.39 c$ & 5919287 & $<0.001$ \\
\hline 1-Octanol & $13.62 \pm 0.93 c$ & $10.03 \pm 1.41 \mathrm{~b}$ & $7.22 \pm 0.31 \mathrm{a}$ & $22.42 \pm 1.21 \mathrm{e}$ & $20.18 \pm 1.51 d$ & 94.268 & $<0.001$ \\
\hline 1-Decanol & $3.35 \pm 0.03 a$ & $3.34 \pm 0.1 \mathrm{a}$ & $3.37 \pm 0.07 a$ & $4.08 \pm 0.22 b$ & $4.37 \pm 0.16 c$ & 41.043 & $<0.001$ \\
\hline Benzyl alcohol & $16109.36 \pm 2149.44 b$ & $15493.54 \pm 727.35 b$ & $9257.79 \pm 1130.95 a$ & $15595.42 \pm 583.54 b$ & $10512.17 \pm 1710.27 a$ & 16.263 & $<0.001$ \\
\hline 2-Phenylethyl alcohol & $\underline{53202.98 \pm 7725.3 a}$ & $\underline{71088.75 \pm 3911.39 c}$ & $\frac{56548.31 \pm}{7924.3 \mathrm{ab}}$ & $\frac{84707.01 \pm}{6389.57 d}$ & $\frac{66573.21 \pm}{6464.95 b c}$ & 10.692 & 0.001 \\
\hline $\begin{array}{l}\text { Total of higher } \\
\text { alcohols }\end{array}$ & $\begin{array}{l}384410.7 \pm \\
18665.87 b\end{array}$ & $\begin{array}{l}372394.87 \pm \\
17414.07 \mathrm{ab}\end{array}$ & $\begin{array}{l}\overline{342891.31} \pm \\
18018.44 \mathrm{a}\end{array}$ & $\begin{array}{l}\overline{471382.19} \pm \\
19896.22 \mathrm{c}\end{array}$ & $\overline{400396.6 \pm 8012.18 b}$ & 24.017 & $<0.001$ \\
\hline Ethyl butanoate & $571.72 \pm 50.3 b$ & $713.84 \pm 21.15 c$ & $424.44 \pm 13.64 a$ & $1127.7 \pm 45.61 d$ & $756.03 \pm 41.07 c$ & 149.829 & $<0.001$ \\
\hline Ethyl hexanoate & $1460.15 \pm 122.94 b$ & $\overline{1343.7 \pm 27.05 b}$ & $1040.55 \pm 53.68 \mathrm{a}$ & $\overline{2512.5 \pm 59.23 d}$ & $\overline{1764.69 \pm 42.62} c$ & 195.674 & $<0.001$ \\
\hline Ethyl heptanoate & $0.74 \pm 0.04 a$ & $0.92 \pm 0.02 b c$ & $0.81 \pm 0.02 \mathrm{ab}$ & $1.32 \pm 0.14 \mathrm{~d}$ & $0.95 \pm 0.06 c$ & 29.501 & $<0.001$ \\
\hline Ethyl octanoate & $2537.79 \pm 179.09 c$ & $1468.4 \pm 64.76 b$ & $1155.54 \pm 47.06 a$ & $4297.72 \pm 222.74 d$ & $2645.28 \pm 140.76 c$ & 211.812 & $<0.001$ \\
\hline Ethyl nonanoate & $1.26 \pm 0.07 \mathrm{a}$ & $1.25 \pm 0.08 \mathrm{a}$ & $1.17 \pm 0.03 \mathrm{a}$ & $1.51 \pm 0.03 \mathrm{c}$ & $1.38 \pm 0.04 \mathrm{~b}$ & 19.368 & $<0.001$ \\
\hline Ethyl decanoate & $553.82 \pm 35.87 b$ & $379.15 \pm 20.11 a$ & $347.83 \pm 19.41 \mathrm{a}$ & $994.85 \pm 14.44 d$ & $664.39 \pm 47.23 c$ & 227.867 & $<0.001$ \\
\hline Ethyl phenylacetate & - & - & $0.18 \pm 0.13 a$ & $0.61 \pm 0.11 \mathrm{~b}$ & $0.64 \pm 0.09 \mathrm{~b}$ & 42.062 & $<0.001$ \\
\hline Ethyl dodecanoate & $36.99 \pm 2.25 b$ & $31.69 \pm 1.39 \mathrm{a}$ & $28.58 \pm 1.79 a$ & $85.49 \pm 4.27 d$ & $54.92 \pm 2.55 c$ & 237.914 & $<0.001$ \\
\hline Isoamyl acetate & $853.42 \pm 115.31 a$ & $1314.52 \pm 108.18 b$ & $837.56 \pm 28.82 a$ & $1241.55 \pm 76.6 b$ & $952.59 \pm 131.04 a$ & 15.303 & $<0.001$ \\
\hline Hexyl acetate & $8.7 \pm 1.64 a$ & $15.09 \pm 2.01 \mathrm{~b}$ & $7.08 \pm 0.73 a$ & $19.86 \pm 2.41 c$ & $15.18 \pm 3.2 \mathrm{~b}$ & 17.667 & $<0.001$ \\
\hline 2-Phenylethyl acetate & $13.42 \pm 2.35 \mathrm{a}$ & $23.3 \pm 3.61 c$ & $18.48 \pm 0.96 b$ & $19.71 \pm 1.82 b c$ & $18.73 \pm 1.77 b$ & 7.25 & 0.005 \\
\hline Ethyl acetate & $\underline{155274.99 \pm 4844.3 d}$ & $\frac{110221.65 \pm}{8925.97 b}$ & $\frac{81197.93 \pm}{3068.01 a}$ & $\frac{163305.87 \pm}{6451.87 d}$ & $\frac{124867.59 \pm}{10648.21 \mathrm{c}}$ & 63.09 & $<0.001$ \\
\hline Methyl octanoate & $6.18 \pm 0.42 d$ & $3.59 \pm 0.14 b$ & $\overline{2.84 \pm 0.08 a}$ & $8.57 \pm 0.37 \mathrm{e}$ & $\overline{5.49 \pm 0.12 c}$ & 219.607 & $<0.001$ \\
\hline Isoamyl octanoate & $5.59 \pm 0.32 c$ & $4.15 \pm 0.13 b$ & $3.53 \pm 0.18 a$ & $11.45 \pm 0.25 \mathrm{e}$ & $6.14 \pm 0.49 d$ & 323.077 & $<0.001$ \\
\hline Isopentyl hexanoate & $5.76 \pm 0.37 c$ & $5.15 \pm 0.13 b$ & $4.06 \pm 0.1 \mathrm{a}$ & $11.07 \pm 0.5 \mathrm{e}$ & $6.57 \pm 0.25 d$ & 232.516 & $<0.001$ \\
\hline Diethyl succinate & $223.58 \pm 29.04 b$ & $153.42 \pm 7.24 \mathrm{a}$ & $134.77 \pm 12.16 \mathrm{a}$ & $525.21 \pm 15.23 d$ & $330.28 \pm 34.85 c$ & 154.746 & $<0.001$ \\
\hline Total of esters & $\begin{array}{l}161554.09 \pm \\
5338.84 d\end{array}$ & $115679.81 \pm 9104.07 b$ & $85205.36 \pm 3158.86 a$ & $\begin{array}{l}174164.99 \pm \\
6658.03 d\end{array}$ & $\begin{array}{l}132090.84 \pm \\
10974.99 c\end{array}$ & 66.736 & $<0.001$ \\
\hline Hexanoic acid & $257.65 \pm 25.86 \mathrm{~cd}$ & $157.49 \pm 83.4 \mathrm{ab}$ & $85.2 \pm 36.87 a$ & $308.71 \pm 15.01 d$ & $191.76 \pm 23.48 b c$ & 11.603 & 0.001 \\
\hline Octanoic acid & $\overline{534.77 \pm 58.38 b}$ & $\overline{472.5 \pm 40.94 \mathrm{ab}}$ & $\overline{388.34 \pm 22.04 a}$ & $\overline{743.82 \pm 78.28 c}$ & $\overline{526.04 \pm 76.17 b}$ & 14.804 & $<0.001$ \\
\hline Decanoic acid & $82.06 \pm 2.65 \mathrm{ab}$ & $91.2 \pm 3.21 \mathrm{~b}$ & $73.91 \pm 6.7 a$ & $120.72 \pm 13.85 \mathrm{~d}$ & $96.7 \pm 6.83 c$ & 15.83 & $<0.001$ \\
\hline Total of volatile acids & $874.48 \pm 85.4 b$ & $721.18 \pm 120.93 a b$ & $547.46 \pm 65.52 a$ & $1173.26 \pm 106.45 c$ & $814.5 \pm 104.79 b$ & 16.357 & $<0.001$ \\
\hline Citronellol & $3.84 \pm 0.40 \mathrm{a}$ & $3.66 \pm 0.41 \mathrm{a}$ & $4.02 \pm 0.48 a$ & $3.68 \pm 0.16 a$ & $3.48 \pm 0.45 a$ & 0.789 & 0.558 \\
\hline Linalool & $\underline{10.83 \pm 0.18 \mathrm{a}}$ & $\underline{11.27 \pm 0.12 \mathrm{~b}}$ & $\underline{11.28 \pm 0.21 \mathrm{~b}}$ & $13.32 \pm 0.08 \mathrm{c}$ & $14.79 \pm 0.11 \mathrm{~d}$ & 862.701 & $<0.001$ \\
\hline$\beta$-Damascenone & $4.56 \pm 0.12 a$ & $5.24 \pm 0.08 b$ & $5.68 \pm 0.05 c$ & $6.38 \pm 0.10 \mathrm{e}$ & $6.01 \pm 0.12 \mathrm{~d}$ & 186.165 & $<0.001$ \\
\hline Geraniol & $\overline{11.89 \pm 0.14 a}$ & $\overline{14.91 \pm 0.05 \mathrm{~b}}$ & $14.99 \pm 0.15 \mathrm{~b}$ & $\overline{17.91 \pm 0.01 \mathrm{c}}$ & $\overline{18.44 \pm 0.05 \mathrm{~d}}$ & 3478.07 & $<0.001$ \\
\hline
\end{tabular}

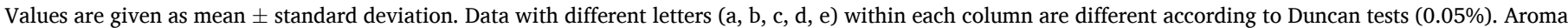
compounds of OVA $>1$ were highlighted; aroma compounds of OVA $>0.1$ were underlined.

below $300 \mathrm{mg} / \mathrm{L}$ gives a pleasant character (Rapp \& Versini, 1995). In this work, the total concentrations of higher alcohols are ranged in $355.39 \mathrm{mg} / \mathrm{L}$ (Concerto/D254) to $453.53 \mathrm{mg} / \mathrm{L}$ (TD12/D254) after ALF and $474.30 \mathrm{mg} / \mathrm{L}$ (Concerto/D254) to $538.77 \mathrm{mg} / \mathrm{L}$ (Prelude/D254) after MLF, respectively. Isoamyl alcohol (fatty and chemical notes), 2, 3-butanediol (fruity and sweet notes), 2-phenylethyl alcohol (flowery and sweet notes), isobutyl alcohol (green and chemical notes), and 1-hexanol (herbaceous note) are the main higher alcohols of the red wines. D254 single fermentation produced a higher concentration of isobutyl alcohol than those of the mixed culture trials. TD12/D254 sequential fermentation had a positive effect on 1-hexanol, isoamyl alcohol, 2,3-butanediol and 2-phenylethyl alcohol formation, with $121.89 \%, 117.96 \%, 125.46 \%$ and $159.21 \%$ increment compared to D254 single fermentation, respectively. In particular, the trials involving 
Table 3

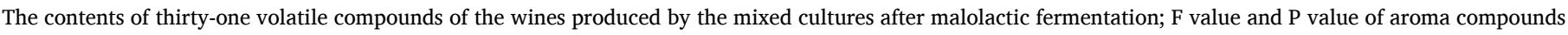
were analyzed by one-way ANOVA.

\begin{tabular}{|c|c|c|c|c|c|c|c|}
\hline Compounds $(\mu \mathrm{g} / \mathrm{L})$ & D254 & Prelude/D254 & Concerto/D254 & TD12/D254 & LT9/D254 & F value & $P$ value \\
\hline 1-Hexanol & $4071.95 \pm 344.94 b c$ & $3268.34 \pm 203.70 \mathrm{a}$ & $3827.52 \pm 31.62 b$ & $4485.96 \pm 116.95 d$ & $4323.28 \pm 31.86 \mathrm{~cd}$ & 19.388 & $<0.001$ \\
\hline Isobutyl alcohol & $\overline{41352.42 \pm 2686.09 c}$ & $\overline{51129.19 \pm 3765.92 \mathrm{~d}}$ & $\underline{32259.97 \pm 194.30 \mathrm{a}}$ & $\frac{39367.64 \pm}{\underline{1755.84 b c}}$ & $\frac{36492.60 \pm}{\underline{2466.33 \mathrm{ab}}}$ & 24.292 & $<0.001$ \\
\hline Isoamyl alcohol & $\frac{215681.1 \pm}{20256.07 a}$ & $\frac{224554.79 \pm}{11140.43 a}$ & $\underline{203915.6 \pm 511.34 a}$ & $\overline{\frac{210011.04}{3871.44 a}}$ & $\overline{\frac{206868.99}{8664.11 a}}$ & 1.6 & 0.249 \\
\hline 2,3-Butanediol & $\frac{\overline{104542.02} \pm}{\underline{1245.21 \mathrm{~b}}}$ & $\frac{\overline{100014.30} \pm}{\underline{2145.28 \mathrm{a}}}$ & $\frac{109945.05 \pm}{\underline{2298.32 c}}$ & $\frac{125553.45}{2893.12 \mathrm{~d}}$ & $\frac{130001.22 \pm}{1986.35 \mathrm{e}}$ & 8008.999 & $<0.001$ \\
\hline 1-Octanol & $14.77 \pm 2.52 b$ & $\overline{5.3 \pm 1.31 a}$ & $14.64 \pm 0.93 b$ & $20.41 \pm 1.16 c$ & $18.13 \pm 1.01 \mathrm{c}$ & 44.171 & $<0.001$ \\
\hline 1-Decanol & $3.8 \pm 0.1 b$ & $3.32 \pm 0.08 \mathrm{a}$ & $4.75 \pm 0.18 d$ & $4.11 \pm 0.06 c$ & $4.14 \pm 0.09 c$ & 67.348 & $<0.001$ \\
\hline Benzyl alcohol & $\underline{40871.41 \pm 3637.23 c}$ & $\frac{33727.83 \pm}{\underline{3265.55 b c}}$ & $\underline{22673.89 \pm 4445.67 a}$ & $\underline{26598.83 \pm 6211.6 \mathrm{ab}}$ & $\frac{28167.54 \pm}{\underline{5945.72 \mathrm{ab}}}$ & 6.373 & 0.008 \\
\hline 2-Phenylethyl alcohol & $\frac{103589.34 \pm}{\underline{4713.43 a}}$ & $\frac{\overline{126069.97} \pm}{\underline{14256.83 a}}$ & $\frac{101657.46 \pm}{17052.45 a}$ & $\frac{91652.91 \pm}{19692.79 a}$ & $\frac{\overline{111974.02} \pm}{24849.71 \mathrm{a}}$ & 1.637 & 0.240 \\
\hline $\begin{array}{l}\text { Total of higher } \\
\text { alcohols }\end{array}$ & $\begin{array}{l}\overline{510126.98} \pm \\
30308.99 \mathrm{ab}\end{array}$ & $\begin{array}{l}\overline{538773.04} \pm \\
25552.72 b\end{array}$ & $\begin{array}{l}\overline{474298.88} \pm \\
22083.78 a\end{array}$ & $\begin{array}{l}\overline{497694.35} \pm \\
25459.16 \mathrm{ab}\end{array}$ & $\begin{array}{l}\overline{517849.92} \pm \\
36531.14 \mathrm{ab}\end{array}$ & 2.125 & 0.152 \\
\hline Ethyl butanoate & $643.7 \pm 68.39 b$ & $757.71 \pm 74.72 \mathrm{c}$ & $495.29 \pm 3.11 \mathrm{a}$ & $1022.21 \pm 53.91 d$ & $806.55 \pm 22.5 c$ & 42.044 & $<0.001$ \\
\hline Ethyl hexanoate & $\overline{1444.11 \pm 161.29 b c}$ & $\overline{1075.65 \pm 146.87 a}$ & $\overline{991.95 \pm 6.07 a}$ & $\overline{1580.09 \pm 133.1 \mathrm{c}}$ & $\overline{1326.24 \pm 37.76 b}$ & 13.693 & $<0.001$ \\
\hline Ethyl heptanoate & $0.94 \pm 0.08 \mathrm{a}$ & $0.89 \pm 0.31 \mathrm{a}$ & $1.01 \pm 0.01 \mathrm{a}$ & $0.85 \pm 0.08 \mathrm{a}$ & $0.87 \pm 0.03 a$ & 0.524 & 0.720 \\
\hline Ethyl octanoate & $4390.88 \pm 448.18 b$ & $2562.01 \pm 340.14 a$ & $2446.16 \pm 51.11 a$ & $4435.78 \pm 363.54 b$ & $4173.12 \pm 31.72 b$ & 33.668 & $<0.001$ \\
\hline Ethyl nonanoate & $2.42 \pm 0.12 \mathrm{a}$ & $3.16 \pm 0.38 b$ & $4 \pm 0.06 c$ & $2.17 \pm 0.19 \mathrm{a}$ & $3.66 \pm 0.07 c$ & 43.862 & $<0.001$ \\
\hline Ethyl decanoate & $1662.56 \pm 136.24 b$ & $1160.95 \pm 137.09 a$ & $1189.34 \pm 42.95 a$ & $1655.69 \pm 93.14 b$ & $1627.76 \pm 13.46 b$ & 21.082 & $<0.001$ \\
\hline Ethyl phenylacetate & $0.5 \pm 0.25 \mathrm{a}$ & $0.35 \pm 0.22 \mathrm{a}$ & $1.39 \pm 0.23 \mathrm{~b}$ & $1.07 \pm 0.28 \mathrm{~b}$ & $1.38 \pm 0.18 \mathrm{~b}$ & 13.403 & 0.001 \\
\hline Ethyl dodecanoate & $118 \pm 8.27 c$ & $97.35 \pm 12.57 \mathrm{ab}$ & $84.57 \pm 5.89 a$ & $106.08 \pm 1.98 \mathrm{bc}$ & $96.2 \pm 3.72 \mathrm{ab}$ & 8.33 & 0.003 \\
\hline Isoamyl acetate & $664.18 \pm 77.64 a$ & $996.02 \pm 119.32 c$ & $756.6 \pm 6.32 \mathrm{ab}$ & $874.53 \pm 61.8 b c$ & $918.66 \pm 25.19 c$ & 10.537 & 0.001 \\
\hline Hexyl acetate & $11.29 \pm 1.42 b$ & $10.12 \pm 1.61 \mathrm{~b}$ & $6.21 \pm 0.15 a$ & $14.48 \pm 1.54 c$ & $16.36 \pm 0.3 c$ & 32.908 & $<0.001$ \\
\hline 2-Phenylethyl acetate & $13.71 \pm 0.86 a$ & $24.22 \pm 2.64 c$ & $20.23 \pm 1.43 b$ & $19.02 \pm 1.49 b$ & $23.43 \pm 0.28 c$ & 21.828 & $<0.001$ \\
\hline Ethyl acetate & $\frac{219574.04 \pm}{\underline{5602.09 c}}$ & $\frac{177375.74 \pm}{15974.58 b}$ & $\frac{125863.43 \pm}{\underline{438.77 a}}$ & $\frac{194228.16 \pm}{10945.22 b}$ & $\frac{183153.69 \pm}{4380.36 b}$ & 41.54 & $<0.001$ \\
\hline Methyl octanoate & $9.4 \pm 1 c$ & $4.43 \pm 0.75 a$ & $\overline{5.16 \pm 0.06 a}$ & $7.64 \pm 0.71 b$ & $\overline{7.15 \pm 0.2 b}$ & 28.257 & $<0.001$ \\
\hline Isoamyl octanoate & $14.08 \pm 1.02 b c$ & $12.29 \pm 1.65 \mathrm{ab}$ & $11.87 \pm 0.48 a$ & $\underline{14.35 \pm 0.86 \mathrm{c}}$ & $\underline{12.72 \pm 0.26 \mathrm{abc}}$ & 3.792 & 0.040 \\
\hline Isopentyl hexanoate & $10.28 \pm 0.89 c$ & $8.6 \pm 1.05 \mathrm{ab}$ & $7.51 \pm 0.1 \mathrm{a}$ & $\overline{10.46 \pm 0.79 c}$ & $9.23 \pm 0.13 b c$ & 8.809 & 0.003 \\
\hline Diethyl succinate & $773.95 \pm 42.88 c$ & $445.75 \pm 33.14 b$ & $284.59 \pm 42.72 \mathrm{a}$ & $446.88 \pm 61.72 b$ & $474.35 \pm 64.27 b$ & 37.422 & $<0.001$ \\
\hline Total of esters & $\begin{array}{l}229334.02 \pm \\
6535.27 d\end{array}$ & $\begin{array}{l}184535.24 \pm \\
16834.02 b\end{array}$ & $132169.29 \pm 437.31 \mathrm{a}$ & $\begin{array}{l}204419.46 \pm \\
11564.55 c\end{array}$ & $\begin{array}{l}192651.38 \pm \\
4533.79 b c\end{array}$ & 40.005 & $<0.001$ \\
\hline Hexanoic acid & $479.19 \pm 50.9 b$ & $355.85 \pm 5.91 \mathrm{ab}$ & $282.35 \pm 37.41 \mathrm{a}$ & $342.6 \pm 141.88 \mathrm{ab}$ & $\underline{360.6 \pm 76.74 a b}$ & 2.555 & 0.104 \\
\hline Octanoic acid & $1224.61 \pm 132.13 \mathrm{~b}$ & $\overline{683.86 \pm 21.64 a}$ & $\overline{796.08 \pm 103.74 a}$ & $\overline{861.05 \pm 255.07 a}$ & $\overline{814.7 \pm 204.02 a}$ & 4.676 & 0.022 \\
\hline Decanoic acid & $151.3 \pm 28.13 \mathrm{~b}$ & $111.07 \pm 7.25 \mathrm{a}$ & $141.23 \pm 5.65 \mathrm{ab}$ & $118.91 \pm 19.04 \mathrm{ab}$ & $115.66 \pm 18.98 \mathrm{a}$ & 2.907 & 0.078 \\
\hline Total of volatile acids & $\overline{1855.1 \pm 198.10 \mathrm{~b}}$ & $\overline{1150.78 \pm 20.27 a}$ & $\overline{1219.66 \pm 146.18 a}$ & $\overline{1322.56 \pm 411.77} \mathrm{a}$ & $\overline{1290.96 \pm 299.47 \mathrm{a}}$ & 3.682 & 0.043 \\
\hline Citronellol & $5.23 \pm 1.14 \mathrm{c}$ & $2.70 \pm 0.32 \mathrm{a}$ & $4.23 \pm 0.58 b c$ & $3.17 \pm 0.31 \mathrm{ab}$ & $3.25 \pm 0.39 \mathrm{ab}$ & 7.752 & 0.004 \\
\hline Linalool & $11.51 \pm 0.04 a$ & $11.54 \pm 0.10 \mathrm{a}$ & $11.48 \pm 0.21 \mathrm{a}$ & $13.62 \pm 0.14 \mathrm{~b}$ & $15.3 \pm 0.12 b$ & 8.892 & 0.002 \\
\hline$\beta$-Damascenone & $2.5 \pm 0.21 \mathrm{a}$ & $\overline{2.28 \pm 0.08 a}$ & $\overline{2.38 \pm 0.13 a}$ & $\overline{3.25 \pm 0.09 b}$ & $\overline{2.49 \pm 0.03 a}$ & 29.143 & $<0.001$ \\
\hline Geraniol & $\overline{11.00 \pm 0.14 a}$ & $\overline{14.21 \pm 0.11 \mathrm{~b}}$ & $\overline{14.86 \pm 0.05 \mathrm{~b}}$ & $\underline{17 \pm 0.01 \mathrm{c}}$ & $\overline{18.74 \pm 0.01 c}$ & 25.875 & $<0.001$ \\
\hline
\end{tabular}

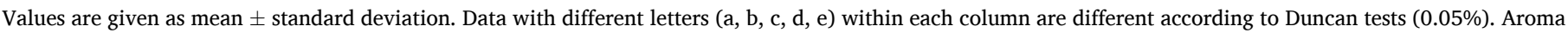
compounds of OVA $>1$ were highlighted; aroma compounds of OVA $>0.1$ were underlined.

T. delbrueckii yeasts produced significantly higher level of 2-phenylethyl alcohol than D254 single fermentation, with 1.59-fold of TD12/D254 and 1.34-fold of Prelude/D254, which was in agreement with the previous data (Canonico et al., 2017; Chen \& Liu, 2016). It should be noticed that the significantly increased levels of higher alcohols $(\mathrm{OAV}>0.1)$ were produced by indigenous $T$. delbrueckii and L. thermotolerans strains compared to commercial non-Saccharomyces strains, respectively, especially in TD12/D254 wine, $30.31 \%$ and $19.16 \%$ increment of isoamyl alcohol and 2-phenylethanol were observed compared to Prelude/D254 wine, which may be due to the different genetic characterizations between indigenous and commercial non-Saccharomyces strains. After MLF, the highest amount of 1-hexanol was obtained in TD12/D254, however, more isobutyl alcohol, isoamyl alcohol, benzyl alcohol and 2-phenylethyl alcohol were produced in the wine of Prelude/D254. The diversities of higher alcohols (expected for isobutyl alcohol) analyzed by $\mathrm{F}$ values of one-way ANOVA in all treatments were decreased when compared to ALF. Those results confirmed that lactic acid bacteria could result in greater modification of high alcohols in wines experienced the mixed starters in ALF (Zhang, Luan, Duan, \& Yan, 2018).

A total of sixteen esters were detected in this study, principally comprising ethyl acetate and ethyl octanoate. The total concentrations of esters are ranged in $85.21 \mathrm{mg} / \mathrm{L}$ (Concerto/D254) to $174.16 \mathrm{mg} / \mathrm{L}$
(TD12/D254) after ALF and $132.17 \mathrm{mg} / \mathrm{L}$ (Concerto/D254) to 229.33 $\mathrm{mg} / \mathrm{L}$ (D254) after MLF, respectively. It is interesting to note that the ester profiles amongst treatments are significantly different $(\mathrm{p}<0.05)$. TD12/D254 was characterized by higher intensities of ethyl esters (OAV $>0.1$ ), including ethyl butanoate, ethyl hexanoate, ethyl octanoate and ethyl decanoate, contributing to the wine with desirable and fruity sensory properties, including banana, strawberry and green apple (Cai et al., 2014). Prelude/D254 was distinguished by the greater abundance of isoamyl acetate, responsible for the banana note. Our results are in agreement with the prior literature that co-fermentation of $T$. delbrueckii and $S$. cerevisiae can produce a high concentration of esters (Renault et al., 2015), however, this property is largely strain-specificity (Loira et al., 2014). The significant divergences of esters also appeared in L. thermotolerans/S. cerevisiae trials, and LT9/D254 produced a higher amount of most esters than Concerto/D254. After MLF, TD12/D254 wine still contained the highest level of ethyl butanoate, ethyl hexanoate and ethyl octanoate, and Prelude/D254 wine had the maximum concentration of isoamyl acetate. Meanwhile, both TD12/D254 and LT9/D254 sequential fermentation favored the production of ethyl butanoate, ethyl hexanoate, ethyl octanoate and ethyl decanoate, especially ethyl octanoate; their levels were increased by $73.14 \%$ and $70.60 \%$ compared to those produced by Prelude/D254 and Concerto/D254, respectively. The different responses were found in isoamyl 
acetate, in which Prelude/D254 produced a 13.89\% higher level than TD12/D254. The similar tendency of esters between the experimental groups after ALF and MLF indicated that non-Saccharomyces strains could significantly influence the content of esters compounds in final wines. Lactic acid bacteria decreased the diversities of the most ester compounds in all treatments after MLF, mainly including ethyl butanoate, ethyl hexanoate, ethyl octanoate and ethyl decanoate.

Volatile fatty acids are formed by yeasts during fatty acid metabolism, which contributes rancid, pungent, fruity, or cheesy odors to wine when above their thresholds (Swiegers \& Pretorius, 2005). Three volatile fatty acids (hexanoic acid, octanoic acid and decanoic acid) were detected in this work, in which TD12/D254 produced the maximum levels of those fatty acids, followed by D254 and LT9/D254. The contents of fatty acids were increased by MLF, and D254 single fermentation produced the highest increment. Contrary to ALF, no significant differences appeared in hexanoic acid and decanoic acid after MLF, suggesting that lactic acid bacteria might have an important impact on fatty acids metabolism in final wines.

Four compounds belonging to the terpene group were identified in this study, i.e., citronellol, linalool, geraniol and $\beta$-damascenone. The content of linalool (sweet and floral note), geraniol (floral note), and $\beta$-damascenone (sweet and floral note) was enhanced by the mixed fermentation when compared to D254 single fermentation. Among them, TD12/D254 and LT9/D254 generated more terpenes than commercial non-Saccharomyces trials. After MLF, $\beta$-damascenone level was significantly decreased, especially in the wine of LT9/D254 (only half of the value of alcoholic fermentation). TD12/D254 had the highest intensity of $\beta$-damascenone, and LT9/D254 resulted in a larger yield of linalool and geraniol in final wines, whose intensities were $33.28 \%$ and 26.11\% higher than those in Concerto/D254, respectively.

\subsection{Multivariate analysis}

To visualize the differences of aroma composition produced by different inoculations, PCAs (principal component analysis) were applied using nineteen main aromatic compounds (OAV>0.1) after ALF (Fig. 3A) and MLF (Fig. 3B). The first and second accounted for $68.1 \%$ (PC1) and $17.9 \%$ (PC2) of the total variation after alcoholic fermentation, and $37.6 \%$ (PC1) and $28.0 \%$ (PC2) after malolactic fermentation, respectively. For ALF, the pure fermentation of D254 was separated by PC2 from the mixed fermentation trials. The wines inoculated of commercial non-Saccharomyces strains located in the negative part of PC1, and the wines of TD12/D254 and LT9/D254 were loaded in the positive part of PC1. The main separated compounds between TD12/D254 and Prelude/D254 was most esters (ethyl butanoate, ethyl octanoate, ethyl hexanoate and ethyl decanoate) and volatile fatty acids (octanoic acid and decanoic acid), whereas in LT9/D254 and Concerto/D254 wines was isoamyl acetate, which further confirmed that the volatiles characteristics of wine inoculated with indigenous and commercial nonSaccharomyces strains were distinct. After MLF, the wine composition was further varied. The wine of D254 pure culture was in the first quadrant, characterized with benzyl alcohol, isoamyl alcohol, hexanoic acid, octanoic acid and decanoic acid. The differences of wine components inoculated with indigenous and commercial non-Saccharomyces strains were clear as well, and principal aroma compounds were 2-phenylethyl alcohol, isobutyl alcohol, isoamyl acetate and ethyl butanoate.

\subsection{Wine odor profile}

An aromatic series of wines could be defined as a group of aroma compounds with similar odor descriptors (Duan et al., 2015; Liu et al., 2016). In this study, six aromatic series were established by the combination of OAVs of a group of active aroma compounds (OAV $>0.1$ ) with similar aroma type to better understand the influence of different non-Saccharomyces strains on wine odor profile, including fruity, floral, sweet, herbaceous, rancid, and solvent (Fig. 4, Supplementary Table 1).
A fruity attribute was prominent in six aromatic series, followed by floral, sweet, herbaceous, solvent, and rancid series. The sequential fermentation trials after ALF had improvement effects on fruity, floral and sweet attributes compared to D254 single fermentation excepted for Prelude/D254 and Concerto/D254 on the fruity aroma. Among them, the wine from TD12/D254 sequential fermentation obtained the highest values of fruity, sweet and floral aroma series due to the increased contents of 2-phenylethyl alcohol, ethyl esters, $\beta$-damascenone and fatty acids. There were no considerable discrepancies in the herbaceous, rancid, and solvent aroma series among different treatments, which are due to the low concentration or high odor threshold of related aroma compounds. After MLF, the divergences of aroma series among different wines were decreased, and TD12/D254 still scored the high aromatic quality of fruity, sweet and floral. These results indicated that TD12/D254 can intensify the pleasant aroma attributes of wine, and confirmed that indigenous strains with excellent enological characteristics have more potential to improve the aroma profiles of regional wines compared to extraneous strains (Nisiotou et al., 2018).

In recent years, co-inoculating non-Saccharomyces strains with $S$. cerevisiae received increasing attention in the winemaking industry because it can produce wine products with distinctive and diversified aroma expected by consumers. To data, there are more than twenty-six non-Saccharomyces strains have been commercialized (Roudil et al., 2020). However, how to choose suitable non-Saccharomyces strains paired with $S$. cerevisiae becomes a difficult issue for winemakers when aims to produce wine products with distinctive and regional characteristics. In previous studies, inoculating autochthonous yeast is strongly recommended (Calabretti et al., 2012). The results of the present study well supported this conclusion. The autochthonous TD12 combined with D254 generated distinct aromatic characteristics (high concentrations of ethyl esters, higher alcohols, linalool and $\beta$-damascenone) in comparison with the pair of commercial strain T. delbrueckii and D254, which was featured by high production of acetate esters (especially isoamyl acetate). Both LT9 and Concerto strains co-fermented with D254 could generate a high amount of lactic acid compared to pure fermentation, but LT9/D254 had obviously higher aroma intensity than the wine produced by Concerto/D254. The lower fermentation rates of Prelude/D254 and Concerto/D254 relative to TD12/D254 and LT9/D254, respectively, verified the previous reports that the commercial strains cannot well adapt to the micro-conditions of the wine region and grape must composition (Rainieri \& Pretorius, 2000), which could partly explain the differences of wines produced by different non-Saccharomyces mixed inoculations. In addition, the diverse genetic characteristics of indigenous and commercial non-Saccharomyces yeasts, although belonging to the same species, are also responsible for the high divergences of wine compositions. Actually, there is a reported study have revealed a wide phenotypic variability existing in $T$. delbrueckii strain, such as volatile acidity $(0.01-0.57 \mathrm{~g} / \mathrm{L})$, glycerol (3.30-6.03 g/L), higher alcohols (56-133 mg/L), esters (3.15-3.45 mg/L) and volatile fatty acids (1.51-3.16 mg/L) (van Breda et al., 2013). Our data well demonstrate these results from the viewing of the mixed inoculated cultures. To explain the different enological and aromatic properties of the same species strains, it is necessary to compare the transcriptional and metabolic profiles under single fermentation and mixed fermentation conditions.

After MLF, the chemical and aromatic compositions of wines produced by different mixed fermentations were further modified. For example, the production of glycerol, acetic acid, lactic acid, higher alcohols, ethyl esters, and volatile fatty acids was enhanced, while acetate esters and $\beta$-damascenone were attenuated compared to after ALF. Meanwhile, the diversity of most aroma compounds between different treatments were decreased after MLF, which attenuated the improvement effects of mixed fermentation compared to the single fermentation of $S$. cerevisiae. These results indicated that the differences of nutrition status in wines after ALF and (or) the interaction of yeast and lactic acid bacteria could further influence the formations of primary and 

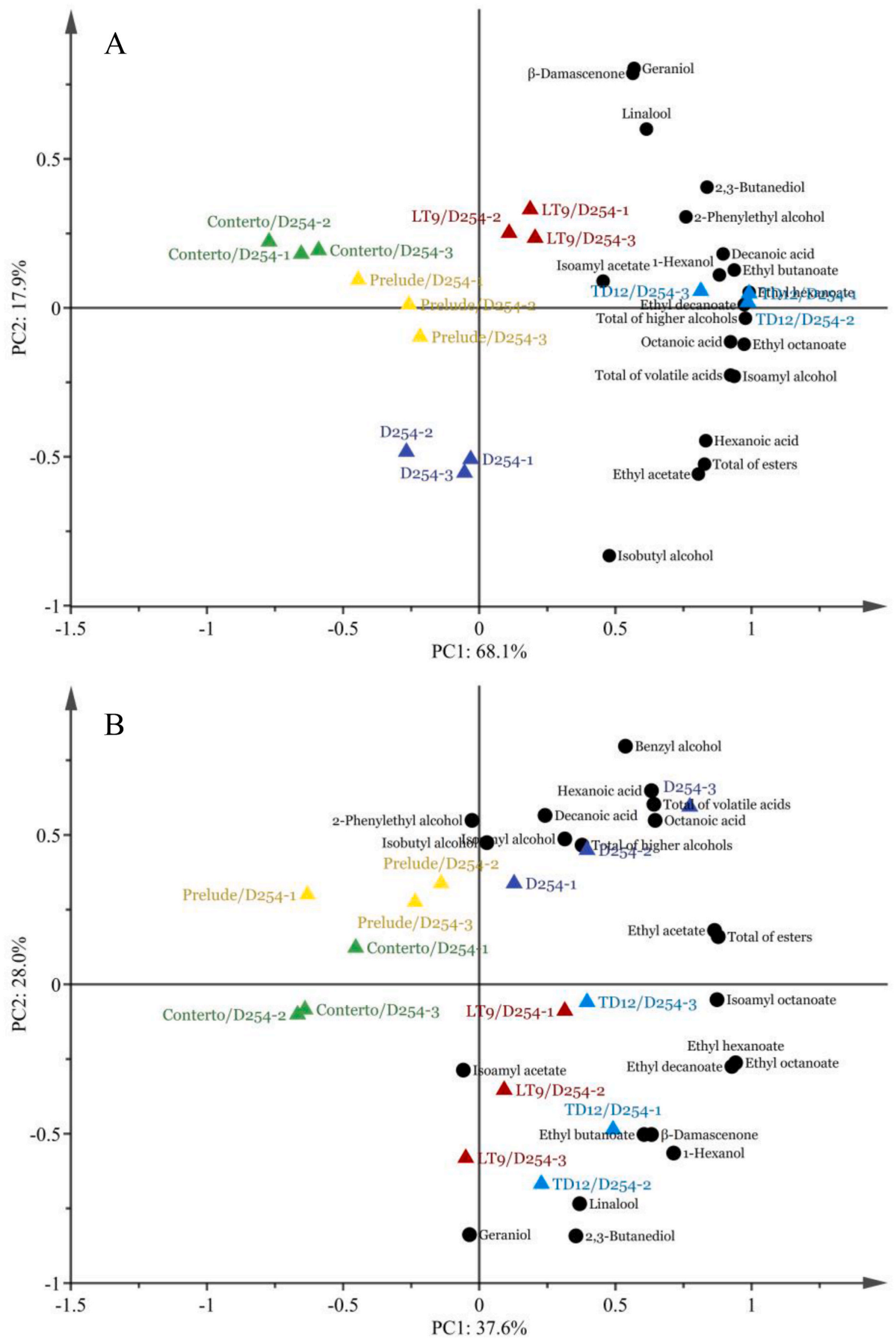

Fig. 3. PCA biplots performed on aromatic compounds $(\mathrm{OAV}>0.1)$ produced by different strains after alcoholic fermentation $(\mathrm{A})$ and malolactic fermentation (B), respectively. 


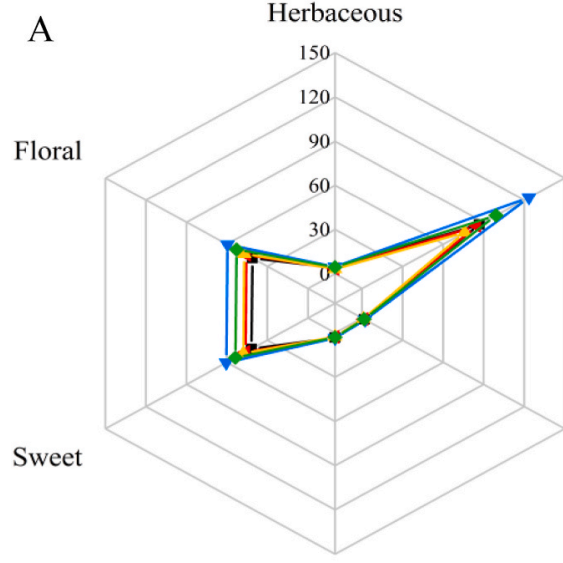

Solvent

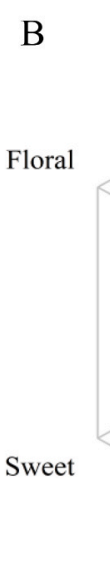

Rancid

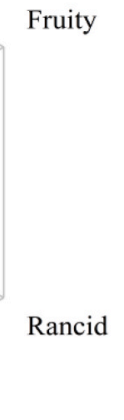

Fig. 4. Odor profile of wines produced by different strains after alcoholic fermentation $(A)$

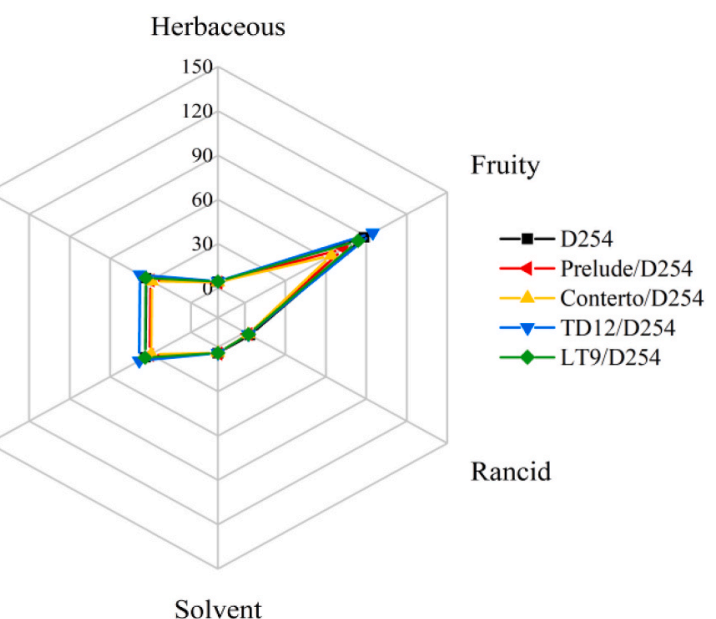

Solvent secondary compounds in final wines, and also suggested that the winemakers should take more considerations when choosing a suitable inoculated combination of yeasts and lactic acid bacteria to improve the quality of regional wines.

\section{Conclusions}

In this work, the enological traits of indigenous strains (T. delbrueckii TD12 and L. thermotolerans LT9) and commercial strains (T. delbrueckii Prelude and L. thermotolerans Concerto) in mixed fermentation with $S$. cerevisiae D254 were evaluated and compared in $20 \mathrm{~L}$ fermenter, respectively. The compositions of wines produced by indigenous and commercial strains were significantly different although belonging to the same species. TD12/D254 was characteristic with high contents of ethyl esters, higher alcohol, linalool, $\beta$-damascenone, and lactic acid, while Prelude/D254 was featured with the highest concentration of acetate esters (mainly isoamyl acetate) and a low amount of acetic acid. LT9/D254 produced more ethyl esters, acetate esters and comparable level of lactic acid relative to Concerto/D254. The divergences of aromatic properties in wines were reduced after MLF, and TD12/D254 still scored high aromatic quality. Our results highlighted the importance of non-Saccharomyces strains in shaping the aromatic quality of wine in mixed fermentation, and suggested that their enological properties are largely strain-specificity, which usually lead to distinctive basic parameters and aroma profiles of the final wines. Thus, more attention should be paid to the strain level when selecting non-Saccharomyces strains in multi-starters wine fermentation. In this regard, the indigenous non-Saccharomyces strains are highly recommended because they not only improve aroma quality and complexity due to better adapt to fermentation conditions, but also impart the wines with typical sensory characteristics specific to each wine area. Due to the red wine quality is determined not only by chemical and aromatic composition, but also by the appearance, structure and taste involving polyphenol substances, more extensive research and large-scale co-fermentation experiments need to be conducted to further confirm the beneficial properties of the both indigenous strains in red wine fermentation, and this is underway in our lab.

\section{Declaration of competing interest}

The authors confirm that there are no conflicts of interest in the manuscript.

\section{Acknowledgments}

This work was supported financially by Ningxia Hui Nationality
Autonomous Region Major Research and Development Project (Item No. 2020BCF01003), the China Agriculture Research System (CARS-29) and China-Macedonia Science and Technology Cooperation and Exchange Project.

\section{Appendix A. Supplementary data}

Supplementary data to this article can be found online at https://doi. org/10.1016/j.fbio.2021.100925.

\section{Author statement}

Designed the experiments: C-QD, V-IP and G-LY. Conducted the experiments: B-QZ and G-LY. Analyzed the experimental data: B-QZ, V-IP and G-LY. Wrote the paper: B-QZ and G-LY.

\section{References}

Balikci, E. K., Tanguler, H., Jolly, N. P., \& Erten, H. (2016). Influence of Lachancea thermotolerans on cv. Emir wine fermentation. Yeast, 33(7), 313-321.

Benito, S. (2018). The impact of Torulaspora delbrueckii yeast in winemaking. Applied Microbiology and Biotechnology, 102(7), 3081-3094.

Binati, R. L., Lemos Junior, W. J. F., Luzzini, G., Slaghenaufi, D., Ugliano, M., \& Torriani, S. (2020). Contribution of non-Saccharomyces yeasts to wine volatile and sensory diversity: A study on Lachancea thermotolerans, Metschnikowia spp. and Starmerella bacillaris strains isolated in Italy. International Journal of Food Microbiology, 318, 108470.

van Breda, V., Jolly, N., \& van Wyk, J. (2013). Characterisation of commercial and natural Torulaspora delbrueckii wine yeast strains. International Journal of Food Microbiology, 163(2), 80-88.

Cai, J., Zhu, B., Wang, Y., Lu, L., Lan, Y., Reeves, M. J., et al. (2014). Influence of prefermentation cold maceration treatment on aroma compounds of Cabernet

Sauvignon wines fermented in different industrial scale fermenters. Food Chemistry, $154,217-229$.

Calabretti, A., La Cara, F., Sorrentino, A., Di Stasio, M., Santomauro, F., Rastrelli, L., et al. (2012). Characterization of volatile fraction of typical Irpinian wines fermented with a new starter yeast. World Journal of Microbiology and Biotechnology, 28(4), 1433-1442.

Canonico, L., Comitini, F., \& Ciani, M. (2017). Torulaspora delbrueckii contribution in mixed brewing fermentations with different Saccharomyces cerevisiae strains. International Journal of Food Microbiology, 259, 7-13.

Casu, F., Pinu, F. R., Fedrizzi, B., Greenwood, D. R., \& Villas-Boas, S. G. (2016). The effect of linoleic acid on the Sauvignon blanc fermentation by different wine yeast strains. FEMS Yeast Research, 16(5). fow050.

Chen, K., Escott, C., Loira, I., Del Fresno, J. M., Morata, A., Tesfaye, W., et al. (2018). Use of non-Saccharomyces yeasts and oenological tannin in red winemaking: Influence on colour, aroma and sensorial properties of young wines. Food Microbiology, 69, 51-63.

Chen, D., \& Liu, S. (2016). Impact of simultaneous and sequential fermentation with Torulaspora delbrueckii and Saccharomyces cerevisiae on non-volatiles and volatiles of lychee wines. Lebensmittel-Wissenschaft und -Technologie- Food Science and Technology, $65,53-61$.

Ciani, M., Comitini, F., Mannazzu, I., \& Domizio, P. (2010). Controlled mixed culture fermentation: A new perspective on the use of non-Saccharomyces yeasts in winemaking. FEMS Yeast Research, 10(2), 123-133. 
Comitini, F., Gobbi, M., Domizio, P., Romani, C., Lencioni, L., Mannazzu, I., et al. (2011). Selected non-Saccharomyces wine yeasts in controlled multistarter fermentations with Saccharomyces cerevisiae. Food Microbiology, 28(5), 873-882.

Domizio, P., Romani, C., Lencioni, L., Comitini, F., Gobbi, M., Mannazzu, I., et al. (2011) Outlining a future for non-Saccharomyces yeasts: Selection of putative spoilage wine strains to be used in association with Saccharomyces cerevisiae for grape juice fermentation. International Journal of Food Microbiology, 147(3), 170-180.

Duan, L. L., Shi, Y., Jiang, R., Yang, Q., Wang, Y. Q., Liu, P. T., et al. (2015). Effects of adding unsaturated fatty acids on fatty acid composition of Saccharomyces cerevisiae and major volatile compounds in wine. South African Journal for Enology and Viticulture, 36(2), 285-295.

Duan, W., Zhu, B., Song, R., Zhang, B., Lan, Y., Zhu, X., et al. (2018). Volatile composition and aromatic attributes of wine made with Vitis vinifera L.cv Cabernet Sauvignon grapes in the Xinjiang region of China: Effect of different commercial yeasts. International Journal of Food Properties, 21(1), 1423-1441.

Erasmus, D. J., Cliff, M., \& Vuuren, H. (2004). Impact of yeast strain on the production of acetic acid, glycerol, and the sensory attributes of icewine. American Journal of Enology and Viticulture, 55(4), 371-378.

Escribano, R., Arenzana, G., L., Portu, J., Garijo, P., Alfaro, L., \& I. (2018). Wine aromatic compound production and fermentative behaviour within different nonSaccharomyces species and clones. Journal of Applied Microbiology, 124(6), 1521-1531.

Gobbi, M., Comitini, F., Domizio, P., Romani, C., Lencioni, L., Mannazzu, I., et al. (2013). Lachancea thermotolerans and Saccharomyces cerevisiae in simultaneous and sequential co-fermentation: A strategy to enhance acidity and improve the overall quality of wine. Food Microbiology, 33(2), 271-281.

Jolly, N. P., Varela, C., \& Pretorius, I. S. (2014). Not your ordinary yeast: nonSaccharomyces yeasts in wine production uncovered. FEMS Yeast Research, 14(2), 215-237.

Kurtzman, C. P., \& Robnett, C. J. (1998). Identification and phylogeny of ascomycetous yeasts from analysis of nuclear large subunit (26S) ribosomal DNA partial sequences. Antonie Van Leeuwenhoek, 73(4), 331-371.

Lan, Y., Qian, X., Yang, Z., Xiang, X., Yang, W., Liu, T., et al. (2016). Striking changes in volatile profiles at sub-zero temperatures during over-ripening of 'Beibinghong' grapes in Northeastern China. Food Chemistry, 212, 172-182.

Li, H., Jiang, D., Liu, W., Yang, Y., Zhang, Y., Jin, C., et al. (2020). Comparison of fermentation behaviors and properties of raspberry wines by spontaneous and controlled alcoholic fermentations. Food Research International, 128, Article 108801.

Liu, S., Laaksonen, O., Kortesniemi, M., Kalpio, M., \& Yang, B. (2018). Chemical composition of bilberry wine fermented with non-Saccharomyces yeasts (Torulaspora delbrueckii and Schizosaccharomyces pombe) and Saccharomyces cerevisiae in pure, sequential and mixed fermentations. Food Chemistry, 266, 262-274.

Liu, P., Lu, L., Duan, C., \& Yan, G. (2016). The contribution of indigenous nonSaccharomyces wine yeast to improved aromatic quality of Cabernet Sauvignon wines by spontaneous fermentation. Lebensmittel-Wissenschaft und-Technologie- Food Science and Technology, 71, 356-363.

Loira, I., Vejarano, R., Bañuelos, M. A., Morata, A., Tesfaye, W., Uthurry, C., et al. (2014). Influence of sequential fermentation with Torulaspora delbrueckii and Saccharomyces cerevisiae on wine quality. Lebensmittel-Wissenschaft und-Technologie- Food Science and Technology, 59(2), 915-922.

Michel, M., Kopecká, J., Meier-Dörnberg, T., Zarnkow, M., Jacob, F., \& Hutzler, M. (2015). Screening for new brewing yeasts in the non-Saccharomyces sector with Torulaspora delbrueckii as model. Yeast, 33, 129-144.
Nisiotou, A., Sgouros, G., Mallouchos, A., Nisiotis, C., Michaelidis, C., Tassou, C., et al. (2018). The use of indigenous Saccharomyces cerevisiae and Starmerella bacillaris strains as a tool to create chemical complexity in local wines. Food Research International, 111, 498-508.

Padilla, B., Gil, J. V., \& Manzanares, P. (2016). Past and future of non-Saccharomyces yeasts: From spoilage microorganisms to biotechnological tools for improving wine aroma complexity. Frontiers in Microbiology, 7, 411.

Porter, T. J., Divol, B., \& Setati, M. E. (2019). Investigating the biochemical and fermentation attributes of Lachancea species and strains: Deciphering the potential contribution to wine chemical composition. International Journal of Food Microbiology, 290, 273-287.

Prior, K. J., Bauer, F. F., \& Divol, B. (2019). The utilisation of nitrogenous compounds by commercial non-Saccharomyces yeasts associated with wine. Food Microbiology, 79, 75-84.

Puertas, B., Jiménez, M. J., Cantos-Villar, E., Cantoral, J. M., \& Rodríguez, M. E. (2017). Use of Torulaspora delbrueckii and Saccharomyces cerevisiae in semi-industrial sequential inoculation to improve quality of Palomino and Chardonnay wines in warm climates. Journal of Applied Microbiology, 122(3), 733-746.

Rainieri, S., \& Pretorius, I. S. (2000). Selection and improvement of wine yeasts. Annals of Microbiology, 50(1), 15-31.

Rapp, A., \& Versini, G. (1995). Influence of nitrogen compounds in grapes on aroma compounds of wines. Developments in Food Science, 1659-1694.

Raymond Eder, M. L., Reynoso, C., Lauret, S. C., \& Rosa, A. L. (2017). Isolation and identification of the indigenous yeast population during spontaneous fermentation of Isabella (Vitis labrusca L.) grape must. Frontiers in Microbiology, 8, 532.

Renault, P., Coulon, J., de Revel, G., Barbe, J., \& Bely, M. (2015). Increase of fruity aroma during mixed T. delbrueckii/S. cerevisiae wine fermentation is linked to specific esters enhancement. International Journal of Food Microbiology, 207, 40-48.

Renault, P., Miot-Sertier, C., Marullo, P., Hernández-Orte, P., Lagarrigue, L., LonvaudFunel, A., et al. (2009). Genetic characterization and phenotypic variability in Torulaspora delbrueckii species: Potential applications in the wine industry. International Journal of Food Microbiology, 134(3), 201-210.

Roudil, L., Russo, P., Berbegal, C., Albertin, W., Spano, G., \& Capozzi, V. (2020). NonSaccharomyces commercial starter cultures: Scientific trends, recent patents and innovation in the wine sector. Recent Patents on Food, Nutrition \& Agriculture, 11(1), 27-39.

Santiago, B. (2018). The impacts of Lachancea thermotolerans yeast strains on winemaking. Applied Microbiology and Biotechnology, 102(16), 6775-6790.

Swiegers, J. H., \& Pretorius, I. S. (2005). Yeast modulation of wine flavor. In Advances in applied microbiology (pp. 131-175). Academic Press.

Vilela, A. (2019). Use of non-conventional yeasts for modulating wine acidity. Fermentatio, 5(1), 27.

van Wyk, N., Grossmann, M., Wendland, J., von Wallbrunn, C., \& Pretorius, I. S. (2019). The whiff of wine yeast innovation: Strategies for enhancing aroma production by yeast during wine fermentation. Journal of Agricultural and Food Chemistry, 67(49), 13496-13505.

Xu, X., Cheng, G., Duan, L., Jiang, R., Pan, Q., Duan, C., et al. (2015). Effect of training systems on fatty acids and their derived volatiles in Cabernet Sauvignon grapes and wines of the north foot of Mt. Tianshan. Food Chemistry, 181, 198-206.

Zhang, B., Luan, Y., Duan, C., \& Yan, G. (2018). Use of Torulaspora delbrueckii cofermentation with two Saccharomyces cerevisiae strains with different aromatic characteristic to improve the diversity of red wine aroma profile. Frontiers in Microbiology, 9, 606. 